\title{
Formation of three-particle clusters in hetero-junctions and MOSFET structures
}

\author{
E. P. Nakhmedov ${ }^{1,2}$ and K. Morawetz ${ }^{1}$ \\ ${ }^{1}$ Max-Planck-Institute for the Physics of Complex Systems, Noethnitzer Str. 38, 01187 Dresden, Germany \\ ${ }^{2}$ Azerbaijan Academy of Sciences, Institute of Physics, H. Cavid 33, Baku, Azerbaijan
}

(November 19, 2018)

\begin{abstract}
A novel interaction mechanism in MOSFET structures and GaAs/AlGaAs hetero-junctions between the zone electrons of the two-dimensional (2D) gas and the charged traps on the insulator side is considered. By applying a canonical transformation, off-diagonal terms in the Hamiltonian due to the trapped level subsystem are excluded. This yields an effective three-particle attractive interaction as well as a pairing interaction inside the $2 \mathrm{D}$ electronic band. A type of BetheGoldstone equation for three particles is studied to clarify the character of the binding and the energy of the three-particle bound states. The results are used to offer a possible explanation of the Metal-Insulator transition recently observed in MOSFET and hetero-junctions.
\end{abstract}

71.10.-w,71.30.+h,73.20.Dx,73.30.+y

Significant advances in low temperature physics are particularly connected with the recent successes in fabricating two-dimensional (2D) electronic structures characterized by high mobility. The formation of the inversion layer of particles on interfaces allows the study of the unusual low-temperature behavior of a $2 \mathrm{D}$ electron gas, as well as the examination of theoretical predictions. Particularly, experimental measurements at very low temperatures, $T \lesssim 2 \mathrm{~K}$, show quite nonstandard results, like the fractional quantum Hall effect ${ }^{1}$ and the Metal-Insulator transition (MIT) $)^{2,3}$, the origin of which is not yet properly understood. It is worthwhile to observe that the fractional quantum Hall effect and MIT occur only under some special regimes where the temperature and the impurity concentration in the samples are very small. On the other hand the observation of such unusual effects only in $G a A s / A l G a A s$ hetero-junctions and $S i$-MOSFET raises the question whether structural peculiarities of these devices are responsible, or rather some fundamental law is observed.

The band structures of GaAs/AlGaAs heterojunctions and of $S i$-MOSFET are well known, and their general features are identical despite their different structures. The inversion of current carriers occurs at the interface of two semiconductors (or insulator/semiconductor) with different band gaps. These semiconductors are exclusively doped by $p$ - and $n$-type impurities in order to get high mobility in the samples. In extremely clean samples, the doping by one type of either acceptors or donors forms single levels in the gap of each semiconductor. In the process of, e.g., electron inversion, the donor centers in $\mathrm{SiO}_{2}$ of the MOSFET structure (or in, $A l_{x} G a_{1-x} A s$ hetero-junction) become positively charged by transferring electrons to the $2 \mathrm{D}$ electronic band. The charged donors are located within a region of at most $\sim 200 \AA^{4}$ from the oxide-silicon interface, and their energy level lies above the Fermi level. Such interfacial charged states act as trap centers for the band electrons. The density of trapped centers in, e.g.,
MOSFET structures is of the order of $10^{9} \mathrm{~cm}^{-24}$, which is considerably smaller than the typical carrier concentration of $\sim 2 \times 10^{11} \mathrm{~cm}^{-2}$. The Coulomb potential of charged traps seems to be screened imperfectly due to their low densities, and the scattering of the band particles on these trap centers may be more essential than the intra-band particle-particle scattering. In this letter we study the effects of the scattering of band electrons on the charged traps.

The Hamiltonian of the model can be written in the form $H=H_{0}+H_{i n t}$, where

$$
H_{0}=\sum_{\mathbf{k}, \sigma} \varepsilon(\mathbf{k}) a_{\mathbf{k}, \sigma}^{+} a_{\mathbf{k}, \sigma}+\sum_{\sigma} \omega_{0} b_{o, \sigma}^{+} b_{o, \sigma}
$$

and

$$
\begin{aligned}
& H_{i n t}=\sum_{\mathbf{k}, \mathbf{q} ; \sigma, \sigma^{\prime}} V(\mathbf{k})\left[a_{\mathbf{k}, \sigma}^{+} a_{-\mathbf{k}+\mathbf{q}, \sigma^{\prime}}^{+} b_{o, \sigma^{\prime}} a_{\mathbf{q}, \sigma}\right. \\
& \left.+a_{\mathbf{q}, \sigma}^{+} b_{o, \sigma^{\prime}}^{+} a_{-\mathbf{k}+\mathbf{q}, \sigma^{\prime}} a_{\mathbf{k}, \sigma}\right] .
\end{aligned}
$$

In Eqs.(1) and (2), $a_{\mathbf{k}, \sigma}^{+}$and $b_{o, \sigma}^{+}\left(a_{\mathbf{k}, \sigma}\right.$ and $\left.b_{o, \sigma}\right)$ are the creation (annihilation) operators for the electrons in the band and in the trapped levels, respectively.

The trap centers are modeled for simplicity as a dispersionless single level. $\epsilon(\mathbf{k})$ and $\omega_{0}$ in Eq.(1) are the energies of the band electrons and of the trap level, respectively. The energy of the trap centers is considered to be larger than the chemical potential $\mu$ of the band electrons, $\omega_{0} \gtrsim \mu$. The first term in the Hamiltonian $H_{\text {int }}$ represents the scattering of two band electrons via the interaction potential $V(\mathbf{k})$ followed by the trapping of one of them by the donor level. The second term represents the scattering of a band electron with a trapped electron (on a donor level) with finally turning both of them into the band.

For temperatures $k T<\left(\omega_{0}-\mu\right)$, the trapping centers contain a definite number of electrons at thermodynamic equilibrium, and the considered trapping mechanism is assumed to be essential. However, the mechanism 
is destroyed by increasing the gate potential, for then the chemical potential $\mu$ reaches the trap level, causing the filling of all trapping centers. A temperature increase also leads to the destruction of the trapping mechanism.

We apply a unitary transformation ${ }^{5}$ with the intention to result in the cancellation of the off-diagonal term, given by Eq.(2). We will show here that this unitary transformation creates a "trap mediated" effective attraction between three electrons in the band ${ }^{6}$. Notice that our approach to the problem is similar to the cancellation of the phonon subsystem in superconductivity, where a canonical transformation yields an effective attraction between electrons, see, e.g., ${ }^{7}$. Let us expand a new Hamiltonian $\widetilde{H}=e^{-i S} H e^{i S}$ in power series of the operator $S=S_{1}+S_{2}+S_{3}+\cdots$. Regrouping the terms of the same order in $H_{\text {int }}$, the conditions which define $S_{i}, i=1,2, \cdots$ are obtained recursively. $S_{1}$ is determined by $H_{\text {int }}-i\left[S_{1}, H_{0}\right]=0$ and leads to

$$
\begin{aligned}
S_{1}= & i \sum_{\mathbf{k}, \mathbf{q} ; \sigma, \sigma^{\prime}} \frac{V_{\mathbf{k}}}{\epsilon_{\mathbf{k}}+\epsilon_{-\mathbf{k}+\mathbf{q}}-\epsilon_{\mathbf{q}}-\omega_{0}} \\
& \times\left[a_{\mathbf{k}, \sigma^{+}}^{+} a_{-\mathbf{k}+\mathbf{q}, \sigma^{\prime}}^{+} b_{o, \sigma^{\prime}} a_{\mathbf{q}, \sigma}-a_{\mathbf{q}, \sigma}^{+} b_{o, \sigma^{\prime}}^{+} a_{-\mathbf{k}+\mathbf{q}, \sigma^{\prime}} a_{\mathbf{k}, \sigma}\right] .
\end{aligned}
$$

The new Hamiltonian $\widetilde{H}$ can now be written in the form $\widetilde{H}=H_{0}-\frac{i}{2}\left[S_{1}, H_{\text {int }}\right]-i\left[S_{2}, H_{0}\right] . \quad S_{2}$ is obtained from the condition that the off-diagonal terms in the Hamiltonian be cancelled. The equation for $\widetilde{H}$ then becomes $\widetilde{H}=H_{0}-\frac{i}{2}\left[S_{1}, H_{\text {int }}\right]_{\text {diag }}$, where the last term contains only diagonal elements. Introducing Eq. (3) into the expression for $\widetilde{H}$ finally yields the effective Hamiltonian

$$
\widetilde{H}=\widetilde{H_{0}}+H_{e-e}+H_{\triangle}
$$

where $\widetilde{H_{0}}, H_{e-e}$ and $H_{\triangle}$ describe the effective oneparticle Hamiltonian, the electron-electron interaction, and the three-particle clustering, respectively. The oneparticle effective Hamiltonian $\widetilde{H_{0}}$ is given by

$$
\begin{aligned}
\widetilde{H_{0}}=H_{0} & -\sum_{\sigma^{\prime}} b_{o, \sigma^{\prime}}^{+} b_{o, \sigma^{\prime}} \sum_{\mathbf{q}, \sigma} \varepsilon_{1}(\mathbf{q}) a_{\mathbf{q}, \sigma}^{+} a_{\mathbf{q}, \sigma} \\
& +\sum_{\mathbf{q} ; \sigma, \sigma^{\prime}} J(\mathbf{q}) b_{o, \sigma}^{+} b_{o, \sigma^{\prime}} a_{\mathbf{q}, \sigma^{\prime}}^{+} a_{\mathbf{q}, \sigma}, \\
\left(\begin{array}{c}
\varepsilon_{1}(\mathbf{q}) \\
J(\mathbf{q})
\end{array}\right)= & \sum_{\mathbf{k}} \frac{1}{\epsilon_{\mathbf{k}}-\epsilon_{-\mathbf{k}+\mathbf{q}}-\epsilon_{\mathbf{q}}-\omega_{0}}\left(\begin{array}{c}
V_{\mathbf{k}}^{2} \\
V_{\mathbf{k}} V_{-\mathbf{k}+\mathbf{q}}
\end{array}\right) .
\end{aligned}
$$

In thermodynamical equilibrium $\left\langle b_{o, \sigma^{\prime}}^{+} b_{o, \sigma}\right\rangle=\delta_{\sigma, \sigma^{\prime}}$, and the one-particle energy is renormalized, $\widetilde{H_{0}}=$ $\sum_{\mathbf{q}, \sigma} \tilde{\varepsilon}(\mathbf{q}) a_{\mathbf{q}, \sigma}^{+} a_{\mathbf{q}, \sigma}$ with $\tilde{\varepsilon}(\mathbf{q})=\varepsilon(\mathbf{q})+\varepsilon_{1}(\mathbf{q})+J(\mathbf{q})$.

The electron-electron interaction Hamiltonian $H_{e-e}$ also contains terms with spin flipping due to the exchange scattering of $2 \mathrm{D}$ electrons on trapped ones. In thermodynamic equilibrium, $H_{e-e}$ has the usual form

$$
\begin{aligned}
H_{e-e}= & \frac{1}{2} \sum_{\mathbf{k}_{\mathbf{1}}, \mathbf{k}_{\mathbf{2}}, \mathbf{q} ; \sigma, \sigma^{\prime}} V_{e-e}^{(e f f)}\left(\mathbf{k}_{\mathbf{1}}, \mathbf{k}_{\mathbf{2}}, \mathbf{q}\right) \\
& \times a_{\mathbf{k}_{\mathbf{1}}, \sigma}^{+} a_{\mathbf{k}_{\mathbf{2}}, \sigma^{\prime}}^{+} a_{\mathbf{k}_{\mathbf{2}}-\mathbf{q}, \sigma^{\prime}} a_{\mathbf{k}_{\mathbf{1}}+\mathbf{q}, \sigma},
\end{aligned}
$$

where the effective two-particle interaction potential $V_{e-e}^{(e f f)}\left(\mathbf{k}_{\mathbf{1}}, \mathbf{k}_{\mathbf{2}}, \mathbf{q}\right)$ appears to be attractive,

$$
\begin{aligned}
& V_{e-e}^{(e f f)}\left(\mathbf{k}_{\mathbf{1}}, \mathbf{k}_{\mathbf{2}}, \mathbf{q}\right)=\frac{V_{\mathbf{k}_{1}-\mathbf{k}_{\mathbf{2}}+\mathbf{q}}^{2}}{\epsilon_{\mathbf{k}_{1}-\mathbf{k}_{2}+\mathbf{q}}+\epsilon_{\mathbf{k}_{2}}-\epsilon_{\mathbf{k}_{1}+\mathbf{q}}-\omega_{0}} \\
& -\frac{V_{\mathbf{k}_{1}} V_{\mathbf{k}_{\mathbf{2}}-\mathbf{q}}}{\epsilon_{\mathbf{k}_{1}}+\epsilon_{\mathbf{q}}-\epsilon_{\mathbf{k}_{1}+\mathbf{q}}-\omega_{0}}-\frac{V_{\mathbf{k}_{1}} V_{\mathbf{k}_{\mathbf{2}}-\mathbf{q}}}{\epsilon_{\mathbf{k}_{2}-\mathbf{q}}+\epsilon_{\mathbf{q}}-\epsilon_{\mathbf{k}_{2}}-\omega_{0}} \\
& +\frac{V_{\mathbf{k}_{1}} V_{\mathbf{q}}+V_{\mathbf{k}_{2}-\mathbf{q}} V_{\mathbf{q}}}{\epsilon_{\mathbf{q}}+\epsilon_{\mathbf{k}_{2}-\mathbf{q}}-\epsilon_{\mathbf{k}_{2}}-\omega_{0}}+\frac{V_{\mathbf{k}_{1}} V_{\mathbf{q}}+V_{\mathbf{k}_{2}-\mathbf{q}} V_{\mathbf{q}}}{\epsilon_{\mathbf{q}}+\epsilon_{\mathbf{k}_{1}}-\epsilon_{\mathbf{k}_{1}+\mathbf{q}}-\omega_{0}} \\
& +\frac{V_{\mathbf{k}_{1}-\mathbf{k}_{\mathbf{2}}+\mathbf{q}}^{2}}{\epsilon_{\mathbf{k}_{1}-\mathbf{k}_{\mathbf{2}}+\mathbf{q}}+\epsilon_{\mathbf{k}_{\mathbf{2}}-\mathbf{q}}-\epsilon_{\mathbf{k}_{1}}-\omega_{0}} .
\end{aligned}
$$

Indeed, the denominator of each term in $V_{e-e}^{(e f f)}$ is negative, owing to the fact that a donor level lies higher than the chemical potential of band electrons.

The third term in the Hamiltonian (4) describes an effective three-particle scattering,

$$
\begin{gathered}
H_{\triangle}=\frac{1}{2} \sum_{\substack{\mathbf{k}_{1}, \mathbf{k}_{2}, \mathbf{q}_{1}, \mathbf{q}_{2} \\
\sigma_{1}, \sigma_{2}, \sigma_{3}}}\left(\frac{V_{\mathbf{k}_{1}} V_{\mathbf{k}_{2}}}{\epsilon_{\mathbf{k}_{1}}+\epsilon_{-\mathbf{k}_{1}+\mathbf{q}_{1}}-\epsilon_{\mathbf{q}_{1}}-\omega_{0}}\right. \\
\left.+\frac{V_{\mathbf{k}_{1}} V_{\mathbf{k}_{2}}}{\epsilon_{\mathbf{k}_{2}}+\epsilon_{-\mathbf{k}_{2}+\mathbf{q}_{2}}-\epsilon_{\mathbf{q}_{2}}-\omega_{0}}\right) \\
\times a_{\mathbf{q}_{1}, \sigma_{1}}^{+} a_{\mathbf{k}_{2}, \sigma_{2}}^{+} a_{-\mathbf{k}_{\mathbf{2}}+\mathbf{q}_{2}, \sigma_{3}}^{+} a_{-\mathbf{k}_{1}+\mathbf{q}_{1}, \sigma_{3}} a_{\mathbf{q}_{2}, \sigma_{2}} a_{\mathbf{k}_{1}, \sigma_{1}} .
\end{gathered}
$$

Using again the condition of $\mu<\omega_{0}$, it is possible to see that the strength of the three-particle interaction is negative, which results in formation of clusters of three electrons.

This effective attraction among three electrons can be understood according to the following physical argument. The proposed mechanism of two-particle interaction with trapping of one of the particles, in contrast to an intraband electron-electron scattering, destroys locally the electro-neutrality of the $2 D$ electron gas. The necessary electro-neutrality in hetero-junctions or in MOSFET's is restored by the ensuing adaption of the height of the Schottky barrier, i.e. by a change in the value of the band bending energy. However, the trapping and releasing processes are so fast that the barrier's height cannot follow. As a result of the trapping of band electrons, a hole appears which acts as an attractive center for other electrons.

The energy level of the trap centers in the above calculation is chosen to be dispersionless for simplicity. However, even in the single level case, the donor center energies depend on the spatial coordinates of the impurities due to the band bending, and therefore become dispersive. Including the dispersion of the trap level does not change qualitatively our results.

We now proceed to show that a three-particle attractive interaction can lead to the formation of a bound state. To this end, we consider for simplicity only the three-particle interaction, and neglect the pairing interaction. The Schrödinger equation for three identical particles in $2 \mathrm{D}$ with a generic interaction potential 
of the form $V\left(\mathbf{r}_{1}-\mathbf{r}_{\mathbf{2}} ; \mathbf{r}_{\mathbf{3}}-\mathbf{r}_{\mathbf{1}} ; \mathbf{r}_{\mathbf{2}}-\mathbf{r}_{\mathbf{3}}\right)$ is written in the following form after introducing the Jacobi coordinates $\mathbf{R}=\frac{1}{3}\left(\mathbf{r}_{1}+\mathbf{r}_{2}+\mathbf{r}_{3}\right), \mathbf{r}=\mathbf{r}_{3}-\mathbf{r}_{1}$ and $\mathbf{z}=\frac{\mathbf{r}_{1}+\mathbf{r}_{3}}{2}-\mathbf{r}_{2}$

$$
\begin{aligned}
& \left\{-\frac{\hbar^{2}}{2 m}\left(\frac{1}{3} \frac{\partial^{2}}{\partial \mathbf{R}^{2}}+2 \frac{\partial^{2}}{\partial \mathbf{r}^{2}}+\frac{2}{3} \frac{\partial^{2}}{\partial \mathbf{z}^{2}}\right)+V\left(\mathbf{z}-\frac{\mathbf{r}}{2} ; \mathbf{r} ; \mathbf{z}+\frac{\mathbf{r}}{2}\right)\right\} \\
& \times \psi(\mathbf{R}, \mathbf{r}, \mathbf{z})=\left(\epsilon+3 \epsilon_{F}\right) \psi(\mathbf{R}, \mathbf{r}, \mathbf{z}),
\end{aligned}
$$

where $\epsilon$ is the three-particle excitation energy measured from the three-particle Fermi level. After excluding the center of mass coordinate $\mathbf{R}$ by expanding $\psi(\mathbf{R}, \mathbf{r}, \mathbf{z})$ in plane waves, $\psi(\mathbf{R}, \mathbf{r}, \mathbf{z})=\sum_{\mathbf{Q}, \mathbf{p}, \mathbf{q}} e^{\frac{i}{\hbar}(\mathbf{Q R}+\mathbf{p r}+\mathbf{q} \mathbf{z})} \phi(\mathbf{p}, \mathbf{q})$, a Bethe-Goldstone-type equation, similar to the equation for Cooper pairs ${ }^{8}$, is obtained,

$$
\begin{aligned}
& \left(\frac{\mathbf{p}^{2}}{m}+\frac{3 \mathbf{q}^{2}}{4 m}-\epsilon-3 \epsilon_{F}\right) \phi(\mathbf{p}, \mathbf{q}) \\
& +\sum_{\mathbf{p}^{\prime}, \mathbf{q}^{\prime}} \tilde{V}\left(\mathbf{p}^{\prime}, \mathbf{q}^{\prime} ; \mathbf{p}, \mathbf{q}\right) \phi\left(\mathbf{p}^{\prime}, \mathbf{q}^{\prime}\right)=0 .
\end{aligned}
$$

The interaction potential $\tilde{V}\left(\mathbf{p}^{\prime}, \mathbf{q}^{\prime} ; \mathbf{p}, \mathbf{q}\right)$ is assumed to be attractive when the energies of the three particles (before the coordinate transformation) lie in a narrow vicinity $\hbar \omega_{0}$ of the Fermi surface $\left(\epsilon_{F}, \epsilon_{F}+\hbar \omega_{0}\right)$. Here $\hbar \omega_{0}$ is a cut-off energy which is comparable to the order of trap level energy measured from the Fermi level. This condition restricts the energies, $\frac{3}{4} \epsilon_{F}<p^{2} / 2 m<\frac{3}{4}\left(\epsilon_{F}+\hbar \omega_{0}\right)$ and $\epsilon_{F}<q^{2} / 2 m<\epsilon_{F}+\hbar \omega_{0}$, of the quasiparticles obtained after coordinates transformation. Therefore, the simplified attractive interaction for a system of linear size $L$ is $\tilde{V}\left(\mathbf{p}^{\prime}, \mathbf{q}^{\prime} ; \mathbf{p}, \mathbf{q}\right)=-\frac{V_{0}}{L^{4}}$ for $\frac{3}{4} \epsilon_{F}<\frac{p^{2}}{2 m}, \frac{p^{\prime 2}}{2 m}<$ $\frac{3}{4}\left(\epsilon_{F}+\hbar \omega_{0}\right), \epsilon_{F}<\frac{q^{2}}{2 m}, \frac{q^{\prime 2}}{2 m}<\epsilon_{F}+\hbar \omega_{0}$, and 0 otherwise.

The following equation for the bound state energy of a three-particle cluster results,

$$
1=\frac{1}{3} V_{0} N^{2} \int_{0}^{\frac{3}{2} \hbar \omega_{0}} d \xi_{1} \int_{0}^{\frac{3}{2} \hbar \omega_{0}} d \xi_{2} \frac{1}{\xi_{1}+\xi_{2}-\epsilon}
$$

where $N$ is the value of the density of electronic states on the Fermi surface. The integration of Eq.(11) gives an equation for $\epsilon$,

$$
\frac{3}{V_{0} N^{2} \hbar \omega_{0}}=3 \ln \frac{6-2 \tilde{\epsilon}}{3-2 \tilde{\epsilon}}-\tilde{\epsilon} \ln \frac{-4 \tilde{\epsilon}(3-\tilde{\epsilon})}{(3-2 \tilde{\epsilon})^{2}},
$$

where $\tilde{\epsilon}=\epsilon / \hbar \omega_{0}$ is the dimensionless excitation energy. The study of Eq. (12) for arbitrary negative values of $\tilde{\epsilon}$ shows that the r.h.s. of this equation is a monotonic and positive function with a maximum value equal to $\ln 8$ at $\tilde{\epsilon}=0$. This imposes a lower restriction on the attractive potential, $V_{0} \geq \frac{1}{\ln 2 N^{2} \hbar \omega_{0}}$. Consequently, for attractive potentials strong enough, Eq.(12) possesses a unique negative solution for $-\tilde{\epsilon} \in(-\infty, 0)$. This implies the existence of three-particle bound states.

In a weak coupling regime, when $-\tilde{\epsilon} \ll 1$, Eq. (12) is simplified to

$$
\frac{-4 \tilde{\epsilon}}{3 e} \ln \frac{-4 \tilde{\epsilon}}{3 e}=\frac{4}{e}\left(\frac{1}{V_{0} N^{2} \hbar \omega_{0}}-\ln 2\right),
$$

the solution of which does not possess a gap-like form for the excitation energy.

In the opposite case of large negative solutions, $-\tilde{\epsilon} \geq 3$, Eq. (12) leads immediately to the following result for the bound energy in the strong coupling regime, $\epsilon=$ $-\frac{3}{4} V_{0}\left(\hbar \omega_{0} N\right)^{2}$, which shows a clear perturbative noncollective behavior.

This discussion shows that molecular clustering rather than a coherent state is realized in the system. The ground state of the system becomes unstable with respect to the three-particle attraction. This seems to lead to molecular type formation with negative energy.

In conclusion, we want to emphasize that it is possible to understand qualitatively the reason of MIT occurring at very low temperatures in GaAs/AlGaAs heterojunctions and $M O S F E T^{9,2}$ in the framework of the formation of three-particle bound states we describe above. The elastic scattering of electrons on impurities at low temperatures, which is characterized by a relaxation time $\tau_{0}$, results in the localization of all electronic states ${ }^{10}$, under the condition that $\hbar \omega_{0}(n)<k T<\hbar / \tau_{0}$, producing an insulating behavior for conductivity. Here $\omega_{0}(n)$ is the trap level energy measured from the Fermi energy, which is a function of the $2 \mathrm{D}$ electron concentration $n$, or the gate potential. On the other hand, in a regime corresponding to $k T<\hbar \omega_{0}(n)<\hbar / \tau_{0}$, which can be reached by varying the electron concentration or the temperature, the formation of three-particles bound states results in the vanishing of the weak localization corrections to conductivity. This is due to the fact that the scattering of the three-particle clusters on the impurities does not lead to quantum interference. Instead, the cluster's wave function accumulates an additional phase by rotation of the cluster in the process of scattering, while the center of mass motion of the cluster is still extended. The expression for the conductivity can be written as

$$
\sigma(T)=\frac{3 e^{2} n_{\Delta} \tau_{0}}{m}+\frac{e^{2} n_{f} \tau_{0}}{m}\left(1-\frac{\hbar}{2 \pi \epsilon_{F} \tau_{0}} \ln \frac{\tau_{i n}(T)}{\tau_{0}}\right),
$$

where the first and the second terms in Eq. (14) correspond to the Drude and the weak localization contributions, ${ }^{11}$, which correspond to three-particles clusters and free band electrons with concentrations of $n_{\Delta}$ and $n_{f}$ respectively. $\tau_{i n}$ is the inelastic scattering time $\tau_{i n}=a T^{-p}$ where $a$ is some constant, $p \geq 2$ and $p=2$ for probable electron-electron scattering mechanism. Notice that a logarithmic temperature dependence of $\sigma$ in the metallic phase has been observed, ${ }^{3}$, in highmobility $n-S i-M O S F E T$ which is in good agreement with our assumption. Observation of the negative lowfield magnetoresistance in the metallic phase also supports an important role of the quantum interference effects in MIT. We neglect in Eq.(14) an additional logarithmic quantum correction due to the electron-electron interactions, ${ }^{11}$, which is responsible for positive magnetoresistance also observed in experiments, ${ }^{2}$. 
Charge conservation allows us to write the total concentration of particles as $n=n_{\Delta}+n_{f}+n_{t}$, where $n_{t}$ is the concentration of trapped electrons, which exponentially decreases with temperature as $n_{t}(T)=$ $n_{t}^{o} \exp \{-\omega(n) / k T\}$ with $n_{t}^{0}$ being the concentration of trapped impurities. Assuming that the clustering occurs at $T=T_{c}(n), n_{\Delta}$ can be expressed near $T_{c}(n)$ as $\frac{n_{\Delta}}{n}=\frac{T_{c}-T}{T_{c}}$. We rewrite Eq. (14) in the form

$$
\frac{\sigma(T)}{\sigma_{0}}=1+2 \frac{T_{c}-T}{T_{c}}-\frac{\hbar T}{\pi \epsilon_{F} \tau_{0} T_{c}} \ln \frac{T^{*}}{T},
$$

where $\sigma_{0}=e^{2} \tau_{0} n / m$ is Drude conductivity, and $T^{*}=$ $\sqrt{a / \tau_{0}}$. In Eq.(15) we neglected the trap level contribution due to $n_{t} \ll n$. Rescaling $T$ by $T^{*}$ as $T / T^{*} \equiv \tau$ for $\tau<1$ and choosing the parameter of randomness $\lambda=\frac{\hbar}{2 \pi \epsilon_{F} \tau_{0}} \lesssim 1$, the unknowing parameter $T_{c} / T^{*} \equiv \tilde{T}_{c}$ in Eq.(15) can be extracted by fitting $\sigma(T) / \sigma_{0}$ to the experimental data. The temperature dependence of $\sigma$ is drawn in Fig. 1 for the best fit parameters $\tilde{T}_{c}(n)$.

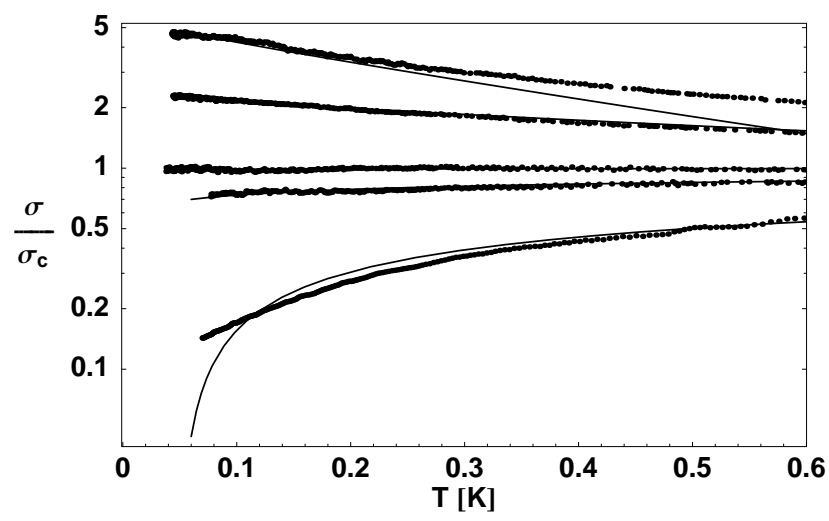

FIG. 1. The temperature dependence of the experimental (dots) conductivity scaled to the critical one $^{12}$. The densities increase from below to above as $n=6.85,7.17,7.25,7.57,7.85 \times 10^{10} \mathrm{~cm}^{-2}$. The lines are theoretical fits according to (15) where the lower three curves are assumed to be undercritical (without $n_{\Delta}$ ) with the fit $T_{c}=3.9,3.6,1.7 \mathrm{~K}$ and $\lambda=0.330,0.078,0.004$ correspondingly and the above two metallic curves are critical (with $n_{\Delta}$ ) with the fit $T_{c}=1.4,0.8 \mathrm{~K}$ and $\lambda=0.48,0.86$.

While the overall fit to MIT is satisfactorily we see deviations on the insulating side. This seems to be due to strong localization effects in these experiments. Effects observed in the weak localization regime, ${ }^{3}$, showing a more pronounced logarithmic behaviour. The value of the conductivity at the critical density $G_{c}$ observed in the two experimets strongly differs from each other: in the strongly disordered case, ${ }^{2}, G_{c} \sim e^{2} / h$, whereas in the weakly disordered case of ${ }^{3} G_{c} \sim 120 e^{2} / h$. Therefore, we think that the conductivity at the critical density $G_{c}$ does not show a universal behavior. It depends on two factors: on the impurity concentration in the $S i$ substrate or in the $2 \mathrm{D}$ electron gas and on the electron concentration (or on the band filling) in the inversion layer. By increasing the band filling in the insulator side of the MIT the three particle clusters appear which weaken the localization tendency in the $2 \mathrm{D}$ electronic system since the scattering off the cluster on the impurities does not lead to the quantum interference effects. At the critical density, the contributions coming from the clustering completely compensate the localization corrections and the conductivity is defined by the value of the residual Drude conductivity, which is temperature independent at low temperature.

Notice that another mechanism for MIT, which is controlled by a temperature-dependent trapped-electron concentration $n_{t}(T)$, has been recently proposed by Altshuler and Maslov ${ }^{13}$. As the comment and reply shows, ${ }^{14}$ this qualitatively correct explanation cannot reproduce quantitative features of the experiment. A critical discussion of different approaches can be found in Ref. ${ }^{2}$.

Although we have not discussed a role of effective pairing governed by Eq.(6) in the Hamiltonian, there was an attempt to interprete the experimental data on MIT as a result of possible superconducting ground state, ${ }^{15}$. It is well known that the effective pairing is suppressed by the order parameter phase fluctuations in 2D systems reducing $T_{S C}$ of the superconducting transition to zero. However, fluctuations of the order parameter modulus above $T_{S C}$ may lead to the metallic phase.

The geometry of the cluster may be either in the form of a triangle with $3 / 2$ and $1 / 2$ total spin, or of a stringlike configuration with $1 / 2$ total spin, when two electrons with antiparallel spins are placed at the same point and the third electron with arbitrary spin is far from them. In the case of $3 / 2$ total spin, a magnetic field parallel to the triangle area does not destroy the cluster, whereas the configurations with $1 / 2$ total spin are destroyed due to the Zeeman effect. In both cases the magnetic field effects are defined by the contributions coming from the quantum localization corrections.

The model of three-particle clustering due to the discussed exchange type of interaction with donor levels seems to be also a favorable candidate for the understanding of the Fractional Quantum Hall effect. The interaction of the band electrons with trap centers effectively leads to a formation of three-particle clusters, see Eq. (8), as well as to the superconducting fluctuations due to effective pairing interactions, Eq. (6). Both mechanisms decrease the ground state energy of the system. Strong magnetic fields in the quantum Hall regime polarize the spins of molecular clusters and a triangular geometry for the cluster is realized due to the Pauli principle. An antisymmetric orbital wave function of the triangular cluster will contain $a b$ initio the Jastrow prefactor. The angular momentum $M=3$ of the cluster provides a natural argument in Laughlin's theory to connect the filling factor $\nu=1 / 3$ of the parent states with the angular momentum $M=3$.

The authors gratefully acknowledge discussions with M. Ameduri, D. Efremov, P. Fulde and K. Maki. 
${ }^{1}$ R. E. Prange and S. M. Girvin, The Quantum Hall Effect (Springer Verlag, Berlin, 1987).

${ }^{2}$ E. Abrahams, S. V. Kravchenko, and M. P. Sarachik, Rev. Mod. Phys. 73, 251 (2001).

${ }^{3}$ V. Pudalov and et al., JETP Lett. 68, 534 (1998).

${ }^{4}$ R. M. Warner and J. B. L. Grung, MOSFET: Theory and Design (Oxford, Oxford, 1999).

${ }^{5}$ J. R. Schrieffer and P. A. Wolff, Phys. Rev. 149, 491 (1966).

${ }^{6}$ E. P. Nakhmedov, (Turkish J. of Physics, Ankara, 1997), Vol. 21, p. 155, Proc. Int. Conf. on Statistical Physics.

${ }^{7}$ C. Kittel, Quantum Theory of Solids (John Wiley, New York, 1987).

${ }^{8}$ P. deGennes, Superconductivity of Metals and Alloys (Addison-Wesley Publishing Company, New York, 1989).

${ }^{9}$ S. V. Kravchenko et al., Phys. Rev.B 50, 8039 (1994).

${ }^{10}$ E. Abrahams, P. W. Anderson, D. C. Licciardello, and T. V. Ramakrishnan, Phys. Rev. Lett. 42, 673 (1979).

${ }^{11}$ P. A. Lee and T. V. Ramakrishnan, Rev. Mod. Phys. 57, 287 (1985).

12 S. V. Kravchenko and T. M. Klapwijk, Phys. Rev. Lett. 84, 2909 (2000).

${ }^{13}$ B. L. Altshuler and D. L. Maslov, Phys. Rev. Lett. 82, 145 (1999).

14 S. V. Kravchenko, M. P. Sarachik, and D. Simonian, Phys. Rev. Lett. 83, 2091 (1999).

${ }^{15}$ P. Phillips and et al., Nature 395, 253 (1998). 